\title{
Electrical conductivity and Hall conductivity of a hot and dense hadron gas in a magnetic field: A relaxation time approach
}

\author{
Arpan Das, ${ }^{1, *}$ Hiranmaya Mishra, ${ }^{1, \dagger}$ and Ranjita K. Mohapatra ${ }^{2, \$}$ \\ ${ }^{1}$ Theory Division, Physical Research Laboratory, Navrangpura, Ahmedabad 380 009, India \\ ${ }^{2}$ Department of Physics, Indian Institute of Technology Bombay, Mumbai 400076, India
}

(Received 19 March 2019; published 22 May 2019)

\begin{abstract}
We estimate the electrical conductivity and the Hall conductivity of a hot and dense hadron gas using the relaxation time approximation for the solution of the Boltzmann transport equation in the presence of an electromagnetic field. We investigate the temperature and the baryon chemical potential dependence of these transport coefficients in the presence of a magnetic field. The explicit calculation is performed within the ambit of the hadron resonance gas model. In general, it is observed that the electrical conductivity decreases in the presence of a magnetic field. While at vanishing magnetic field the electrical conductivity decreases monotonically with temperature, in the presence of a magnetic field the same shows a nonmonotonic behavior with a peak. The Hall conductivity, on the other hand, shows a nonmonotonic behavior with respect to the dependence on a magnetic field as well as with temperature. We argue that for a pair plasma (particle-antiparticle plasma) where $\mu_{B}=0$, Hall conductivity vanishes. Only for a nonvanishing baryon chemical potential, Hall conductivity has a nonzero value. We also estimate the electrical conductivity and the Hall conductivity as a function of the center of mass energy along the freeze-out curve as may be relevant for relativistic heavy-ion collision experiments.
\end{abstract}

DOI: 10.1103/PhysRevD.99.094031

\section{INTRODUCTION}

Transport coefficients of strongly interacting matter created in the relativistic heavy-ion collision experiments are of great importance for a comprehensive understanding of the hot and dense QCD (quantum chromodynamics) medium produced in these experiments. Experimental data and theoretical models give a strong hint about the formation of quark-gluon plasma (QGP) in the initial stage of heavy-ion collisions and its subsequent hadronization, at the Relativistic Heavy Ion Collider (RHIC) and the Large Hadron Collider (LHC). In the dissipative relativistic hydrodynamical model of the hot and dense medium as well as for transport simulations, which are being used to describe the evolution of the strongly interacting matter in heavy-ion collisions, transport coefficients, e.g., shear and bulk viscosity, etc., play an important role. In fact, it has been shown that a small shear viscosity to entropy ratio $(\eta / s)$ is necessary to explain the flow data, in the context of

\footnotetext{
*arpan@prl.res.in

${ }^{\dagger}$ hm@prl.res.in

ranjita@iitb.ac.in
}

Published by the American Physical Society under the terms of the Creative Commons Attribution 4.0 International license. Further distribution of this work must maintain attribution to the author(s) and the published article's title, journal citation, and DOI. Funded by SCOAP. hydrodynamical modeling for the evolution of the QGP medium subsequent to a heavy-ion collision [1-3]. The smallness of $\eta / s$ and its connection with the lower bound for the same, $\eta / s=\frac{1}{4 \pi}$ using gauge gravity duality (AdS/CFT correspondence) has motivated a large number of investigations in understanding the behavior of transport coefficients from a microscopic theory [3]. The bulk viscosity $\zeta$ also plays a significant role in the dissipative hydrodynamics describing the QGP evolution [4-12]. The bulk viscosity encodes the conformal measure $(\epsilon-3 P) / T^{4}$ of the system and lattice QCD simulations show a nonmonotonic behavior for both $\eta / s$ and $\zeta / s$ near the critical temperature $T_{c}$ [6-12]. In the case of noncentral heavy-ion collisions, due to the collision geometry, a large magnetic field is also expected to be produced. The magnitude of the produced magnetic field at the initial stages in these collisions is expected to be rather large, at least of the order of several $m_{\pi}^{2}[13,14]$. Since the strength of the magnetic field is of the hadronic scale, the effect of the magnetic field on the QCD medium can be significant. This prompts a deeper understanding of the QGP, as well as the subsequent hadronic medium in a magnetic field. The strong magnetic field produced in relativistic heavy-ion collision experiments, along with the presence of deconfined non-Abelian QCD matter, brings some exciting possibilities specifically to different $C P$ violating effects such as the chiral magnetic effect and chiral vortical effect [15]. Magnetohydrodynamic simulations which incorporate the large scale behavior of the 
thermal medium in the presence of a dynamical electromagnetic field in a self-consistent manner have been used to study the flow coefficient of strongly interacting matter $[16,17]$. A phenomenological manifestation of a magnetic field requires that the magnetic field survive for at least a few Fermi proper time. Evolution of the electromagnetic field in a conducting plasma is intimately related to the electrical conductivity $\sigma^{e l}$ of the medium [16-34]. Different approaches like perturbative QCD and different effective models, etc., have been used to estimate various transport coefficients for the QCD matter [35-64]. The other transport coefficient that plays a significant role in the hydrodynamical evolution at nonzero baryon densities is the thermal conductivity $[65,66]$.

In the present work, we investigate the electrical and the Hall conductivity of the hot and dense hadron gas produced in the subsequent evolution of QGP, in heavy-ion collisions. The Hall effect is the production of an induced electric current transverse to the electric field and to an applied magnetic field (perpendicular to the electric field) in a conducting medium. The Hall effect is a manifestation of the diffusion of electric charge perpendicular to the electric field. Necessary requirements of the Hall current are the presence of mobile charge carriers and an external electric and magnetic field perpendicular to each other. The electric field and magnetic field produced in heavy-ion collisions can have such configurations $[67,68]$ and, therefore, it is natural to investigate the Hall effect in strongly interacting matter. Indeed, in the presence of a magnetic field the Hall conductivity for the QGP medium has been recently investigated in Ref. [69].

In the present investigation, we study the electrical and Hall conductivity for the hot and dense hadronic matter in a magnetic field using the hadron resonance gas model (HRGM) within the framework of the relaxation time approximation (RTA). It may be noted that in the usual condensed matter systems, e.g., semiconductors, the Hall effect requires applied electric and magnetic fields perpendicular to each other and in these cases either the electrons or the holes are the dominant charge carriers [70]. Furthermore, in the context of electron-ion plasma, the mobilities of the positive and negative charge carriers are different, which can give rise to a net Hall current. On the other hand, for pair plasma (e.g., electron positron plasma) due to the opposite gyration of the positive and negative charge carriers, the net Hall current vanishes [71-73]. This will be similar in quark-gluon plasma (QGP) with a zero baryon chemical potential. However, the situation is different at a finite baryon chemical potential, when the numbers of baryons and antibaryons are different. At a finite baryon chemical potential, unequal numbers of positive and negative charge carriers can generate a net Hall current. At a finite baryon chemical potential, only the net baryons contribute to the Hall current and the mesonic contribution to the Hall current exactly cancels with the opposite Hall current due to its antiparticle. A baryon-rich strongly interacting medium is expected to form at heavy-ion collision experiments at the Facility for Antiproton and Ion Research (FAIR) at Darmstadt [74] and in the Nuclotron-Based Ion Collider Facility (NICA) at Dubna [75]. In these cases, a thermalized strongly interacting medium is expected to be produced at finite baryon density, which is electrically charged. Keeping the above motivation in mind, we calculate the electrical conductivity and Hall conductivity of a hadron resonance gas in a magnetic field within the kinetic theory framework. Let us note that the large magnetic field produced in a heavy-ion collision can be sustained in the QGP medium with finite electrical conductivity. One would expect, however, at later times when the quark hadron transition takes place during the course of evolution, that the magnetic field may not be as strong as in the QGP phase. In the present approach therefore we attempted to estimate the transport coefficients where the phase space and the single particle energies are not affected by a magnetic field through Landau quantization as in Ref. [69]. On the other hand, the effect of a magnetic field in the medium enters through the cyclotron frequency of the individual hadrons.

The hadron resonance gas (HRG) model describes the hadronic phase of the strongly interacting medium created in heavy-ion collisions, at chemical freeze-out [76,77]. This model has been extensively and successfully used to reproduce the experimental result of the thermal abundance of different particle ratios in heavy-ion collisions [78]. In the simplest case when the strange and nonstrange particles freeze out in the same manner, the HRG model has only two parameters: temperature $(T)$ and baryon chemical potential $\left(\mu_{B}\right)$. It has been shown that in the presence of narrow resonances, the thermodynamics of the interacting gas of hadrons can be approximated by the noninteracting gas of hadrons and its resonances $[79,80]$. Because of this phenomenological fact, the thermodynamics of a strongly interacting hadronic system can be easily studied using noninteracting hadrons and their resonances, where the interactions between different hadrons are encoded as the resonances. Due to this simplified noninteracting quasiparticle description, the HRG model has been well explored regarding thermodynamics $[81,82]$, conserved charge fluctuations [83-88], and transport coefficients for hadronic matter [19,21,22,36-60]. Some improvements have also been made in the ideal HRG model, e.g., the HRG model including excluded volume $[60,89]$. We would like to mention here that, although the electrical conductivity has been well explored in the HRG model, the Hall conductivity and the effect of a magnetic field on electrical and Hall conductivity have not been studied earlier for a hadronic system. The present investigation is a first step in that direction.

This paper is organized as follows. In Sec. II we introduce the formalism to estimate electrical conductivity 
and Hall conductivity using kinetic theory within the relaxation time approximation. In Sec. III we briefly discuss the HRG model and estimate the relaxation time within the same model. In Sec. IV we present and discuss the results for electrical and Hall conductivity. Finally we summarize our work with an outlook in the conclusion section.

\section{BOLTZMANN EQUATION IN RELAXATION TIME APPROXIMATION}

The relativistic Boltzmann transport equation (RBTE) of a charged particle of a single species in the presence of an external electromagnetic field can be written as [69]

$$
p^{\mu} \partial_{\mu} f(x, p)+e F^{\mu \nu} p_{\nu} \frac{\partial f(x, p)}{\partial p^{\mu}}=\mathcal{C}[f],
$$

where $F^{\mu \nu}$ is the electromagnetic field strength tensor, $e$ is the electric charge of the particle, and $\mathcal{C}[f]$ is the collision integral. In the RTA, the collision integral can be written as

$$
\mathcal{C}[f] \simeq-\frac{p^{\mu} u_{\mu}}{\tau}\left(f-f_{0}\right) \equiv-\frac{p^{\mu} u_{\mu}}{\tau} \delta f
$$

where $u_{\mu}$ is the fluid four-velocity that in the local rest frame is $(1, \overrightarrow{0})$ and $\tau$ is the relaxation time which determines the timescale for the system to relax towards the equilibrium state characterized by the distribution function $f_{0}$. In the relaxation time approximation, the underlying assumption is that the system is slightly away from equilibrium and then it relaxes towards equilibrium with the timescale $\tau$. In the relaxation time approximation, the external electromagnetic field cannot be very large with respect to the characteristic scale of the thermal system; hence, we are not considering the Landau quantization of the charged particles in a magnetic field. This assumption is plausible in the context of a hadronic medium where the strength of the magnetic field is relatively smaller with respect to the initial large magnetic field produced in heavy-ion collisions. In this work we have considered the Boltzmann distribution function, which has been widely used for the hadron resonance gas model. The equilibrium distribution function satisfies

$$
\frac{\partial f_{0}}{\partial \vec{p}}=\vec{v} \frac{\partial f_{0}}{\partial \epsilon}, \quad \frac{\partial f_{0}}{\partial \epsilon}=-\beta f_{0}, \quad f_{0}=g e^{-\beta\left(\epsilon \pm \mu_{B}\right)},
$$

where the single particle energy is $\epsilon(p)=\sqrt{\vec{p}^{2}+m^{2}}, g$ is the degeneracy, $\mu_{B}$ is the baryon chemical potential, and $\beta=1 / T$ is the inverse of temperature. Using Eq. (2), the Boltzmann equation (1) can be recast as

$$
\frac{\partial f}{\partial t}+\vec{v} \cdot \frac{\partial f}{\partial \vec{r}}+e[\vec{E}+\vec{v} \times \vec{B}] \cdot \frac{\partial f}{\partial \vec{p}}=-\nu\left(f-f_{0}\right),
$$

where $\nu=1 / \tau$. In the case of a uniform and static medium, both $f$ and $f_{0}$ are independent of time and space [69]. In such a case, Eq. (4) becomes

$$
-e[\vec{E}+\vec{v} \times \vec{B}] \frac{\partial f}{\partial \vec{p}}=\nu\left(f-f_{0}\right) .
$$

Without loss of generality, we take $\vec{E}=E \hat{x}$ and $\vec{B}=B \hat{z}$. For this representation of the electric field and magnetic field, Eq. (5) can be rearranged as

$$
\begin{gathered}
\left(\nu-e B\left(v_{x} \frac{\partial}{\partial p_{y}}-v_{y} \frac{\partial}{\partial p_{x}}\right)\right) f(p) \\
=\nu f_{0}(p)-e E \frac{\partial}{\partial p_{x}} f_{0}(p) .
\end{gathered}
$$

In order to solve Eq. (6), we take the following ansatz of the distribution function $f(p)$ [69]:

$$
f(p)=f_{0}-\frac{1}{\nu} e \vec{E} \cdot \frac{\partial f_{0}(p)}{\partial \vec{p}}-\vec{\Xi} \cdot \frac{\partial f_{0}(p)}{\partial \vec{p}} .
$$

Using the ansatz given in Eq. (7), we can simplify Eq. (6):

$$
\begin{aligned}
& \frac{e B}{\nu} e E \frac{v_{y}}{p}-e B\left(v_{x} \Xi_{y}-v_{y} \Xi_{x}\right) \frac{1}{p} \\
& +\nu\left(\Xi_{x} \frac{p_{x}}{p}+\Xi_{y} \frac{p_{y}}{p}+\Xi_{z} \frac{p_{z}}{p}\right)=0 .
\end{aligned}
$$

Equation (8) should be satisfied for any value of the velocity; hence, we get $\Xi_{z}=0$. Comparing the coefficient of $v_{y}$ and $v_{x}$ on both sides of Eq. (8), one obtains

$$
\omega_{c}\left(\frac{e E}{\nu}\right)+\omega_{c} \Xi_{x}+\nu \Xi_{y}=0
$$

and

$$
-\omega_{c} \Xi_{y}+\nu \Xi_{x}=0
$$

In the above equation, $\omega_{c}=e B / \epsilon(p)$ is the cyclotron frequency of the charged particle. Solving Eqs. (9) and (10) for $\Xi_{x}$ and $\Xi_{y}$, one obtains

$$
\Xi_{x}=-e E \frac{\omega_{c}^{2}}{\nu\left(\nu^{2}+\omega_{c}^{2}\right)}, \quad \Xi_{y}=-e E \frac{\omega_{c}}{\omega_{c}^{2}+\nu^{2}} .
$$

Using Eq. (11), the ansatz for the distribution function $f(p)$ given in Eq. (7) can be simplified as, with $p=|\vec{p}|$, 


$$
\begin{aligned}
f(p) & =f_{0}-e E \frac{p_{x}}{p} \frac{\partial f_{0}}{\partial p} \frac{\nu}{\nu^{2}+\omega_{c}^{2}}+e E \frac{p_{y}}{p} \frac{\partial f_{0}}{\partial p} \frac{\omega_{c}}{\omega_{c}^{2}+\nu^{2}} \\
& =f_{0}-e E v_{x}\left(\frac{\partial f_{0}}{\partial \epsilon}\right) \frac{\nu}{\nu^{2}+\omega_{c}^{2}}+e E v_{y}\left(\frac{\partial f_{0}}{\partial \epsilon}\right) \frac{\omega_{c}^{2}}{\omega_{c}^{2}+\nu^{2}} .
\end{aligned}
$$

Electric current is given by [69]

$j^{i}=e \int \frac{d^{3} p}{(2 \pi)^{3}} v^{i} \delta f=\sigma^{i j} E_{j}=\sigma^{e l} \delta^{i j} E_{j}+\sigma^{H} \epsilon^{i j} E_{j}$,

where $\epsilon_{i j}$ is the antisymmetric $2 \times 2$ unity tensor, with $\epsilon_{12}=-\epsilon_{21}=1$. Then the electrical and the Hall conductivity can be identified as

$$
\begin{aligned}
& \sigma^{e l}=e^{2} \int \frac{d^{3} p}{(2 \pi)^{3}} v_{x}^{2}\left(-\frac{\partial f_{0}}{\partial \epsilon}\right) \frac{\nu}{\nu^{2}+\omega_{c}^{2}}, \\
& \sigma^{H}=e^{2} \int \frac{d^{3} p}{(2 \pi)^{3}} v_{y}^{2}\left(-\frac{\partial f_{0}}{\partial \epsilon}\right) \frac{\omega_{c}}{\nu^{2}+\omega_{c}^{2}} .
\end{aligned}
$$

Assuming an isotropic system, the electrical conductivity and the Hall conductivity can be expressed as

$$
\begin{aligned}
\sigma^{e l} & =\frac{e^{2}}{3 T} \int \frac{d^{3} p}{(2 \pi)^{3}} \frac{p^{2}}{\epsilon^{2}} \frac{\nu}{\nu^{2}+\omega_{c}^{2}} f_{0} \\
& =\frac{e^{2}}{3 T} \int \frac{d^{3} p}{(2 \pi)^{3}} \frac{p^{2}}{\epsilon^{2}} \frac{1 / \tau}{(1 / \tau)^{2}+\omega_{c}^{2}} f_{0}, \\
\sigma^{H} & =\frac{e^{2}}{3 T} \int \frac{d^{3} p}{(2 \pi)^{3}} \frac{p^{2}}{\epsilon^{2}} \frac{\omega_{c}}{\nu^{2}+\omega_{c}^{2}} f_{0} \\
& =\frac{e^{2}}{3 T} \int \frac{d^{3} p}{(2 \pi)^{3}} \frac{p^{2}}{\epsilon^{2}} \frac{\omega_{c}}{(1 / \tau)^{2}+\omega_{c}^{2}} f_{0} .
\end{aligned}
$$

It is important to note that, in the absence of a magnetic field, the expression for the electrical conductivity as given in Eq. (16) exactly matches with the standard expression for the same obtained in relaxation time approximation $[22,90]$. In this work we have used thermal averaged relaxation time. Electrical and Hall conductivity of a single species can be extended for a system that consists of multiple charge particle species. In this case,

$$
\begin{aligned}
\sigma^{e l} & =\sum_{i} \frac{e_{i}^{2} \tau_{i}}{3 T} \int \frac{d^{3} p}{(2 \pi)^{3}} \frac{p^{2}}{\epsilon_{i}^{2}} \frac{1}{1+\left(\omega_{c i} \tau_{i}\right)^{2}} f_{0}, \\
\sigma^{H} & =\sum_{i} \frac{e_{i}^{2} \tau_{i}}{3 T} \int \frac{d^{3} p}{(2 \pi)^{3}} \frac{p^{2}}{\epsilon_{i}^{2}} \frac{\omega_{c i} \tau_{i}}{1+\left(\omega_{c i} \tau_{i}\right)^{2}} f_{0},
\end{aligned}
$$

where $e_{i}, \tau_{i}$, and $\omega_{c i}$ are electric charge, thermal averaged relaxation time, and cyclotron frequency of the $i$ th charged particle species. From Eqs. (18) and (19) it is clear that in a pair plasma the particle and antiparticle contribute to the electrical conductivity in the same manner, but in the case of the Hall conductivity their behavior is exactly opposite. Once the relaxation time $\tau$ and cyclotron frequency is known for each species, the electrical conductivity and Hall conductivity can be estimated using Eqs. (18) and (19). In this work we model the hadronic matter using the hadron resonance gas model and estimate relaxation time as well as conductivities.

\section{HADRON RESONANCE GAS MODEL}

The thermodynamic potential of a gas of hadrons and resonances at finite temperature $(T)$ and baryon chemical potential $\left(\mu_{B}\right)$ can be written as [51]

$$
\begin{aligned}
\log Z\left(\beta, \mu_{B}, V\right)= & \int d m\left(\rho_{M}(m) \log Z_{b}\left(m, V, \beta, \mu_{B}\right)\right. \\
& \left.+\rho_{B}(m) \log Z_{f}\left(m, V, \beta, \mu_{B}\right)\right),
\end{aligned}
$$

where $V$ is the volume and $T=1 / \beta$ is the temperature of noninteracting pointlike hadrons and their resonances. The total partition function is composed of the partition functions of free mesons $\left(Z_{b}\right)$ and baryons $\left(Z_{f}\right)$ with mass $m$. Moreover, $\rho_{B}$ and $\rho_{m}$ denote the spectral functions of free bosons (mesons) and fermions (baryons), respectively. The hadron properties are encoded in the spectral densities. Once the spectral density is specified, taking derivatives of the logarithm of the partition function as given in Eq. (20), with respect to the thermodynamic parameters $\mathrm{T}, \mu_{B}$, and the volume $V$, various thermodynamic quantities can be calculated. In this investigation we have considered the HRG model by taking all the hadrons and their resonances below a certain mass cutoff $\Lambda$ to estimate the thermodynamic potential. This is achieved by taking the spectral density $\rho_{B / M}(m)$ of the following form,

$$
\rho_{B / M}(m)=\sum_{i}^{M_{i}<\Lambda} g_{i} \delta\left(m-M_{i}\right) .
$$

In Eq. (21), $M_{i}$ and $g_{i}$ are the masses and the corresponding degeneracy of the known hadrons and their resonances, respectively. It is important to mention that HRG with a discrete particle spectrum is very appealing because of its simple structure; however, it can explain lattice QCD data for a trace anomaly only up to temperature $\sim 130 \mathrm{MeV}$ [91]. Along with the discrete particle spectrum if one also includes the Hagedron spectrum then lattice QCD data for a QCD trace anomaly up to $T \sim 160 \mathrm{MeV}$ [91] can be reproduced. For details of the thermodynamics of the HRG model, see, e.g., Ref. [76].

The relaxation time of particle $a$ having momentum $p_{a}$ and energy $E_{a}$ is defined as $[38,90]$ 


$$
\tau_{a}^{-1}\left(E_{a}\right)=\sum_{b, c, d} \int \frac{d^{3} p_{b}}{(2 \pi)^{3}} \frac{d^{3} p_{c}}{(2 \pi)^{3}} \frac{d^{3} p_{d}}{(2 \pi)^{3}} W(a, b \rightarrow c, d) f_{b}^{0},
$$

where $W(a, b \rightarrow c, d)$ is the transition rate which can be expressed in terms of the transition amplitude $\mathcal{M}$ in the following way:

$W(a, b \rightarrow c, d)=\frac{(2 \pi)^{4} \delta\left(p_{a}+p_{b}-p_{c}-p_{d}\right)}{2 E_{a} 2 E_{b} 2 E_{c} 2 E_{d}}|\mathcal{M}|^{2}$.

In the center of mass frame, the relaxation time $\left(\tau_{a}\right)$ or equivalently interaction frequency $\left(\omega_{a}\right)$ can be written as

$$
\tau_{a}^{-1}\left(E_{a}\right) \equiv \omega_{a}\left(E_{a}\right)=\sum_{b} \int \frac{d^{3} p_{b}}{(2 \pi)^{3}} \sigma_{a b} v_{a b} f_{b}^{0} .
$$

Here $\sigma_{a b}$ is the total scattering cross section for the process $a\left(p_{a}\right)+b\left(p_{b}\right) \rightarrow c\left(p_{c}\right)+d\left(p_{d}\right)$ and $v_{a b}$ is the relativistic relative velocity between particle $a$ and $b$,

$$
v_{a b}=\frac{\sqrt{\left(p_{a} \cdot p_{b}\right)^{2}-m_{a}^{2} m_{b}^{2}}}{E_{a} E_{b}} .
$$

In this work we shall be considering the energy averaged relaxation time. One can obtain the energy-independent relaxation time $\tau^{a}$ by averaging the relaxation time $\tau^{a}\left(E_{a}\right)$ over the distribution function $f_{a}^{0}\left(E_{a}\right)[38,92]$,

$$
\tau_{a}^{-1}=\frac{\int f_{a}^{0} \tau_{a}^{-1}\left(E_{a}\right) d E_{a}}{\int f_{a}^{0} d E_{a}} .
$$

Using Eqs. (24) and (26), the energy averaged relaxation time $\left(\tau_{a}\right)$ can be given as [60]

$$
\tau_{a}^{-1}=\sum_{b} n_{b}\left\langle\sigma_{a b} v_{a b}\right\rangle,
$$

where $n_{b}$ and $\left\langle\sigma_{a b} v_{a b}\right\rangle$ represent number density and thermal averaged cross section, respectively. The thermal averaged cross section for the scattering process $a\left(p_{a}\right)+$ $b\left(p_{b}\right) \rightarrow c\left(p_{c}\right)+d\left(p_{d}\right)$ is given as [93]

$$
\left\langle\sigma_{a b} v_{a b}\right\rangle=\frac{\int d^{3} p_{a} d^{3} p_{b} \sigma_{a b} v_{a b} f_{a}^{0}\left(p_{a}\right) f_{a}^{0}\left(p_{b}\right)}{\int d^{3} p_{a} d^{3} p_{b} f_{a}^{0}\left(p_{a}\right) f_{a}^{0}\left(p_{b}\right)} .
$$

Assuming hard sphere (of radius $r_{h}$ ) scattering for the cross section $\left(\sigma=4 \pi r_{h}^{2}\right)$ and Boltzmann distribution function $f_{a}^{0}\left(p_{a}\right)=e^{-\left(E_{a} \pm \mu_{a}\right) / T}$, the thermal averaged cross section becomes

$$
\left\langle\sigma_{a b} v_{a b}\right\rangle=\frac{\sigma \int d^{3} p_{a} d^{3} p_{b} v_{a b} e^{-E_{a} / T} e^{-E_{b} / T}}{\int d^{3} p_{a} d^{3} p_{b} e^{-E_{a} / T} e^{-E_{b} / T}}
$$

Note that in the above equation the chemical potential dependence gets canceled from the numerator and denominator. This is a consequence of the Boltzmann approximation for the equilibrium distribution function. After changing the momentum integration in the above equation to the center of mass energy variable $(\sqrt{s})$, we get

$$
\begin{aligned}
& \int d^{3} p_{a} d^{3} p_{b} v_{a b} e^{-E_{a} / T} e^{-E_{b} / T} \\
& =2 \pi^{2} T \int d s \sqrt{s}\left(s-4 m^{2}\right) K_{1}(\sqrt{s} / T)
\end{aligned}
$$

and

$$
\int d^{3} p_{a} d^{3} p_{b} e^{-E_{a} / T} e^{-E_{b} / T}=\left(4 \pi m^{2} T K_{2}(m / T)\right)^{2} .
$$

Thus the thermal averaged cross section can be written as

$$
\left\langle\sigma_{a b} v_{a b}\right\rangle=\frac{\sigma}{8 m^{4} T K_{2}^{2}(m / T)} \int_{4 m^{2}}^{\infty} d s \sqrt{s}\left(s-4 m^{2}\right) K_{1}(\sqrt{s} / T) .
$$

Here $\sqrt{s}$ is the center of mass energy; $K_{1}$ and $K_{2}$ are modified Bessel functions of first order and second order, respectively. When the particles are of different species, then the above equation can be written as

$$
\begin{aligned}
\left\langle\sigma_{a b} v_{a b}\right\rangle= & \frac{\sigma}{8 T m_{a}^{2} m_{b}^{2} K_{2}\left(m_{a} / T\right) K_{2}\left(m_{b} / T\right)} \\
& \times \int_{\left(m_{a}+m_{b}\right)^{2}}^{\infty} d s \times \frac{\left[s-\left(m_{a}-m_{b}\right)^{2}\right]}{\sqrt{s}} \\
& \times\left[s-\left(m_{a}+m_{b}\right)^{2}\right] K_{1}(\sqrt{s} / T),
\end{aligned}
$$

where $\sigma=4 \pi r_{h}^{2}$ is the total scattering cross section for the hard sphere. It is important to mention that while the cross section $\sigma$ is independent of both temperature and baryon chemical potential, the thermal averaged cross section $\langle\sigma v\rangle$ in general can depend on temperature $(T)$ and chemical potential $\mu_{B}$ arising from the distribution functions. However, the Boltzmann approximation $\langle\sigma v\rangle$ is independent of $\mu_{B}$ [93]. After evaluating the thermal averaged relaxation time using Eq. (27) for each species, we estimate the electrical conductivity and the Hall conductivity of the hot and dense hadron gas using Eqs. (18) and (19), respectively.

\section{RESULTS AND DISCUSSION}

As mentioned earlier, for the hadron resonance gas model, we consider here all the hadrons and their resonances up to an upper cutoff in mass $\Lambda$ which we take as $\Lambda=2.6 \mathrm{GeV}$ as listed in Ref. [94]. For a detailed list of particles we refer to Appendix A of Ref. [95]. The other 


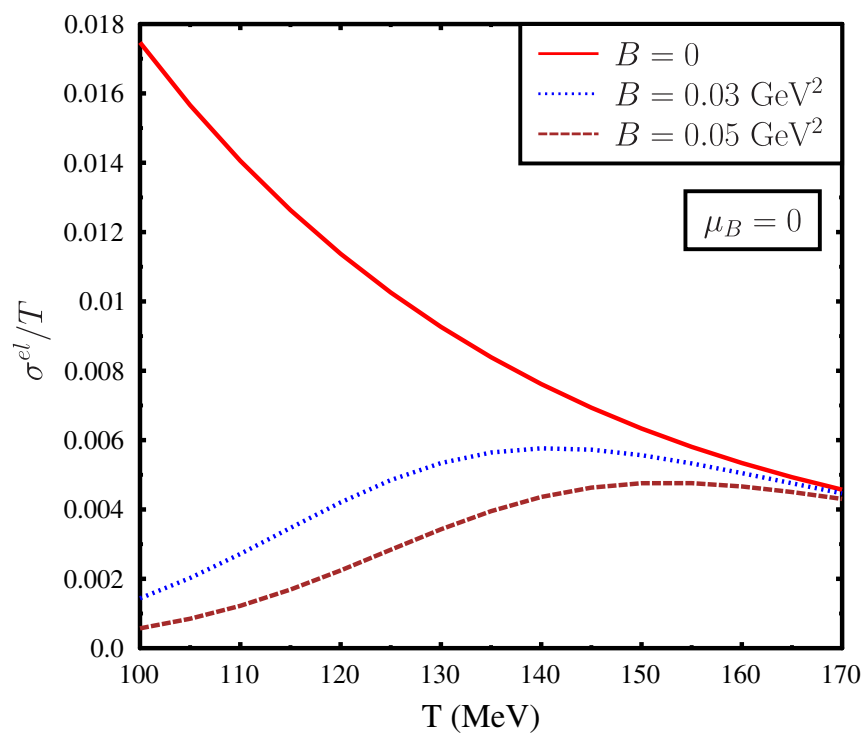

FIG. 1. Variation of normalized electrical conductivity $\left(\sigma^{e l} / T\right)$ with temperature $(T)$ for different values of the magnetic field $(B)$ at zero baryon chemical potential. The red solid line represents the $B=0$ case; the blue dotted line and brown dashed line represent $B=0.03 \mathrm{GeV}^{2}$ and $B=0.05 \mathrm{GeV}^{2}$, respectively. With increasing magnetic field, $\sigma^{e l} / T$ decreases due to larger diffusion of charged particles transverse to the electric and magnetic field.

parameter that enters in the estimation of the relaxation time calculation is the radii of the hard spheres. We have considered a uniform radius of $r_{h}=0.5 \mathrm{fm}$ for all the mesons and baryons $[60,96]$. With this set of parameters, we have estimated the electrical conductivity and the Hall conductivity using Eqs. (18) and (19) as a function of temperature $(T)$ and baryon chemical potential $\left(\mu_{B}\right)$ for different values of the magnetic field $(B)$.

In Fig. 1 we show the variation of electrical conductivity $\sigma^{e l}$ with temperature $(T)$ for different values of the magnetic field $(B)$ at vanishing baryon chemical potential $\left(\mu_{B}\right)$. For a vanishing magnetic field $(B=0)$, the behavior of $\sigma^{e l} / T$ is similar to the previous results; e.g., see Ref. [19]. As may be observed from Fig. 1, $\sigma^{e l} / T$ decreases monotonically with temperature at $B=0$. This can be associated with the increase of randomness of the system with a larger collision rate leading to a smaller relaxation time. We point out here that the dominant contribution to the electrical conductivity arises from the charged pions due to the small mass of the pions as compared to that of other hadrons. Thus the monotonic decrease of $\sigma^{e l} / T$ is due to the decrease of the relaxation time of pions with increasing temperature. It is important to mention that the $1 / T^{2}$ factor in $\sigma^{e l} / T$, as shown in Eq. (18), also affects the variation of electrical conductivity with temperature.

For a nonvanishing magnetic field, the behavior of $\sigma^{e l} / T$ is very different as compared to the $B=0$ counterpart.
Firstly, it is observed that with an increase in the magnetic field strength the electrical conductivity decreases. This decrease in electrical conductivity with the magnetic field can be understood physically. At zero magnetic field, the electric current is along the direction of the electric field. However, at finite magnetic field, charges also diffuse transverse to both the electric and magnetic field, due to the Lorentz force, giving rise to a reduced current along the direction of the electric field. This is also reflected in the expression for electrical conductivity as in Eq. (18). The effect of the magnetic field lies in the cyclotron frequency for the charged hadrons and occurs in the denominator in Eq. (18), leading to a suppression of electrical conductivity. We would like to point out here, however, that small magnetic field effects arising from Landau quantization for the charged hadrons can be neglected and hence the relaxation time arising from the scattering of the hadrons is independent of the magnetic field. The total magnetic field dependence in the expression for electrical conductivity arises only from its dependence on the cyclotron frequency $\left(\omega_{c}\right)$.

Secondly, the temperature dependence of $\sigma^{e l} / T$ at finite magnetic field, as shown in Fig. 1, is rather nontrivial with a nonmonotonic structure in contrast to the monotonic decrease of its $B=0$ counterpart. For a nonzero magnetic field, $\sigma^{e l}$ first increases with temperature and finally decreases. This behavior is mainly due to the combined effect of relaxation time and magnetic field, as can be seen from Eq. (18). With increasing temperature, thermal averaged relaxation time decreases. Hence the numerator of Eq. (18) decreases. But for nonzero magnetic fields, $\left(1+\left(\omega_{c} \tau\right)^{2}\right)$ in the denominator also decreases with temperature. This interplay between $\tau$ and $\omega_{c} \tau$ decides the behavior of $\sigma^{e l}$ with temperature. It is easy to see that for large $\tau$ (low temperature) $\sigma^{e l} \sim \frac{1}{\omega_{c}^{2} \tau}$, leading to an increasing behavior of electrical conductivity with temperature. At higher temperature with a small relaxation time, $\sigma^{e l} \sim \tau$, leading to a decrease in electrical conductivity at higher temperature. Also note that $\sigma_{e l} / T \propto 1 / T^{2}$, as can be seen in Eq. (18), and higher temperature suppresses $\sigma_{e l} / T$. It is important, however, to note that due to the combination of $\omega_{c} \tau$ in Eq. (18), the mass of the particles explicitly enters in the electrical conductivity, apart from the same also in the distribution function. Hence at finite magnetic field contributions of the heavier particles may not be negligible.

Next we consider the variation of normalized electrical conductivity with temperature $(T)$ for various values of baryon chemical potential $\left(\mu_{B}\right)$ at zero magnetic fields as shown in Fig. 2. It is clear from this figure that with increasing chemical potential $\left(\mu_{B}\right)$ electrical conductivity decreases. For the range of $\mu_{B}$ considered here, the contribution to the electrical conductivity from the charged hadrons is dominated by the charged pions, similar to the case with vanishing chemical potential. At finite chemical 


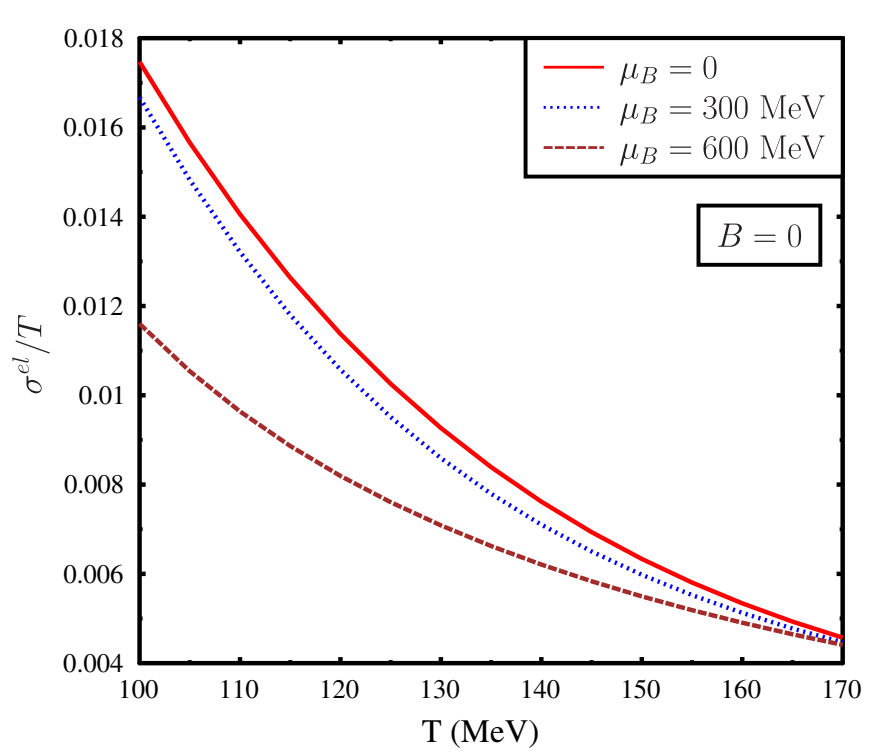

FIG. 2. Variation of normalized electrical conductivity $\sigma^{e l} / T$ with temperature $T$ for different values of baryon chemical potential $\mu_{B}$ at zero magnetic field. The red solid line represents $\mu_{B}=0$ case. The blue dotted and brown dashed lines represent electrical conductivity at finite baryon chemical potential with $\mu_{B}=300 \mathrm{MeV}$ and $\mu_{B}=600 \mathrm{MeV}$, respectively. In the case of zero magnetic field, with increasing $\mu_{B}$ normalized electrical conductivity, $\sigma^{e l} / T$ decreases predominantly due to a decrease in the relaxation time of pions.

potential the pion relaxation time decreases with $\mu_{B}$ due to scattering with the baryons, mostly from the nucleons. One would have naively expected the nucleon contribution to the electrical conductivity to increase with $\mu_{B}$, which will lead to an increase in the total electrical conductivity due to the $\mu_{B}$-dependent distribution function in the expression of electrical conductivity. However, this increase of the baryonic contribution to the electrical conductivity is not enough to compensate for the decreasing contribution arising from pions, at least for the chemical potential considered in the present investigation. This leads to a decrease of the total electrical conductivity with an increase in the baryon chemical potential at vanishing magnetic field.

Next we discuss the variation of normalized electrical conductivity $\sigma^{e l} / T$ with temperature $(T)$ in the presence of a magnetic field and for different values of the baryon chemical potential $\left(\mu_{B}\right)$. This is shown in Fig. 3. Unlike the vanishing magnetic field case, it is seen that $\sigma^{e l} / T$ increases with the baryon chemical potential. This behavior can be understood as follows. At finite magnetic field the contributions of the mesons to the electrical conductivity further decrease due to the larger cyclotron frequency as compared to baryons, apart from the decrease in the relaxation time with the increase in $\mu_{B}$. On the other hand, the contributions of the baryons are enhanced at finite chemical potential. For the chemical potential considered

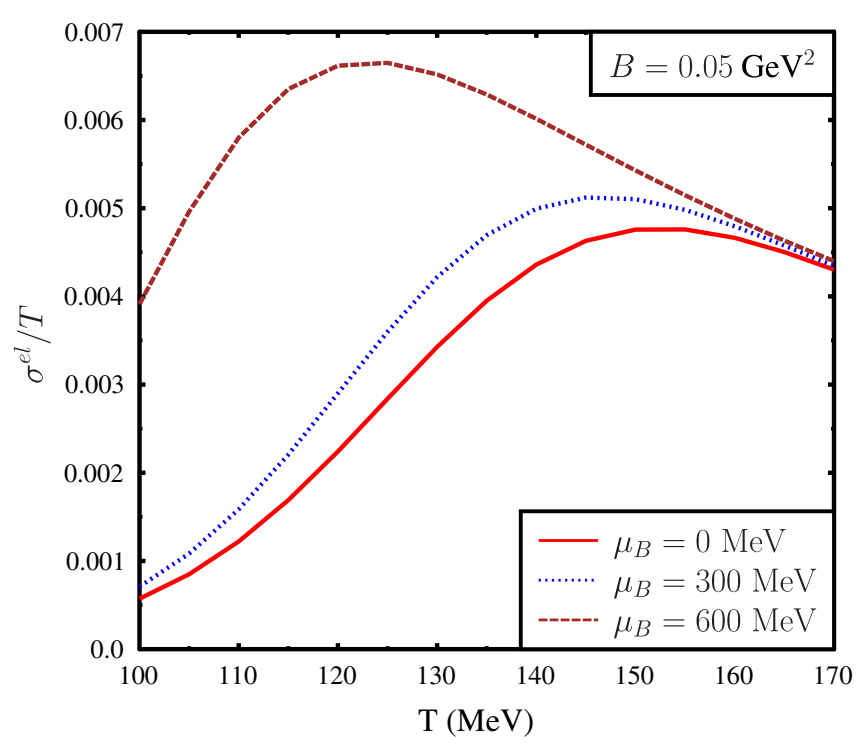

FIG. 3. Variation of normalized electrical conductivity $\sigma^{e l} / T$ with temperature for different values of baryon chemical potential $\mu_{B}$ at $B=0.05 \mathrm{GeV}^{2}$. The red solid line represents $\mu_{B}=0$ result. The blue dotted line and brown dashed line represent $\mu_{B}=$ $300 \mathrm{MeV}$ and $\mu_{B}=600 \mathrm{MeV}$ results, respectively.

here, the contributions of the baryons become larger compared to those of mesons, leading to an increase in electrical conductivity with the baryon chemical potential. This has been explicitly shown in Fig. 4, where we have shown the contributions from baryons and mesons separately for $\mu_{B}=0,300$, and $600 \mathrm{MeV}$ in Figs. 4(a), 4(b), and 4 (c) respectively.

Next, we discuss Hall conductivity in a hadronic gas within the HRG model. In Fig. 5, we show the variation of Hall conductivity with temperature $(T)$ for different values of the magnetic field at finite baryon chemical potential $\mu_{B}=100 \mathrm{MeV}$. Let us note that due to the opposite gyration of the particles and the antiparticles in a magnetic field, the mesonic contribution to the Hall conductivity gets exactly canceled out. Hence, it is only the baryons which contribute to the Hall conductivity at finite baryon chemical potential. It may be observed in Fig. 5 that for low temperature the Hall conductivity decreases with the increase in the magnetic field, while for higher temperature the Hall conductivity increases with the magnetic field. This can be understood from the expression of Hall conductivity in Eq. (19). At low temperature since the relaxation time is larger, the integrand in Hall conductivity $\sim \frac{1}{\omega_{c} \tau}\left(\omega_{c}=\frac{e B}{\epsilon}\right)$, which explains the suppression of Hall conductivity with an increasing magnetic field. On the other hand, at high temperature with a smaller relaxation time the integrand $\sim \omega_{c} \tau$, which explains the increase in the Hall conductivity with increasing magnetic field. Further, it is also interesting to observe that for a fixed value of the magnetic field, the normalized Hall conductivity first 

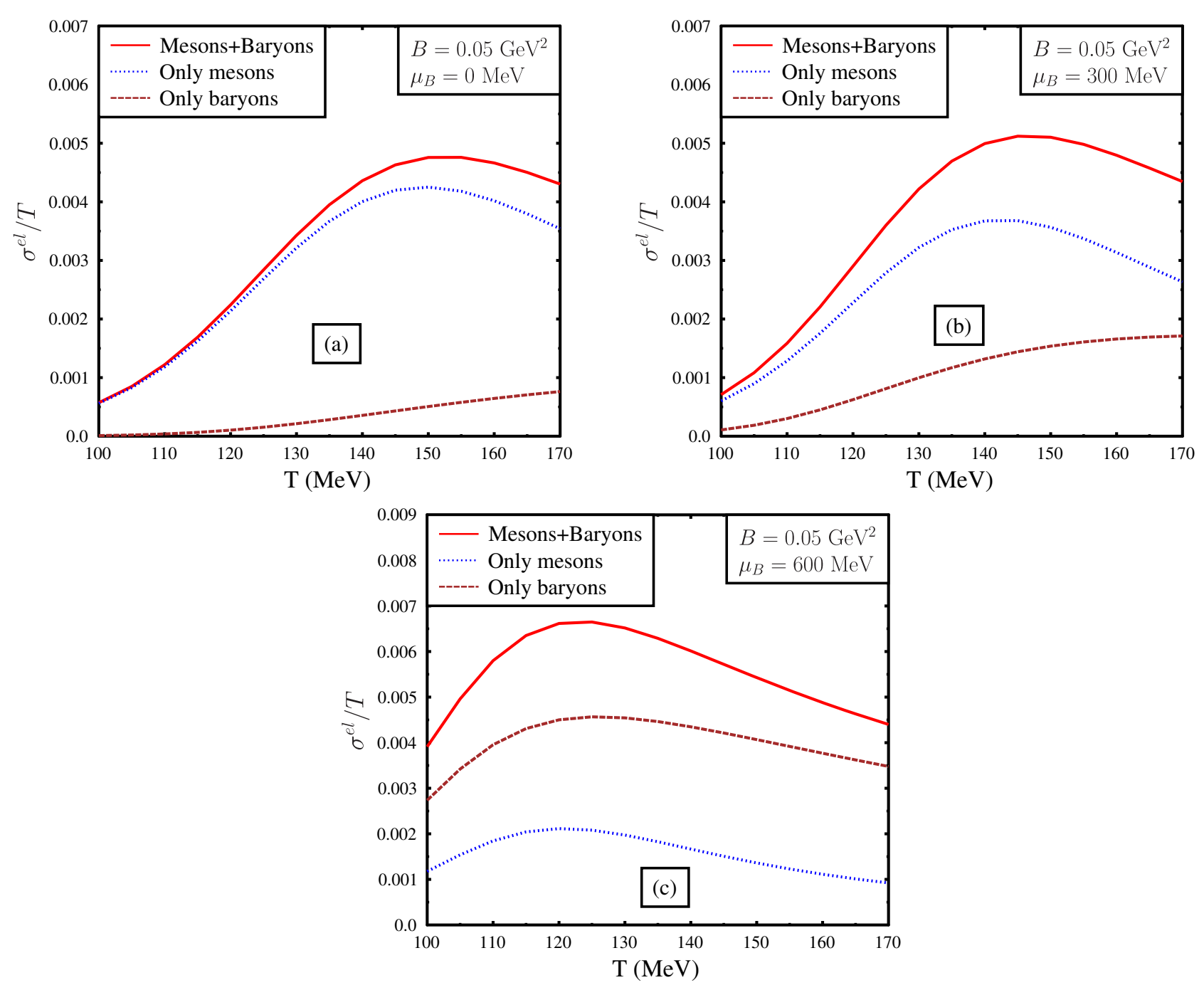

FIG. 4. Variation of normalized electrical conductivity $\sigma^{e l} / T$ (red solid line) with temperature for different values of baryon chemical potential $\mu_{B}$ at $B=0.05 \mathrm{GeV}^{2}$ : (a) $\mu_{B}=0 \mathrm{MeV}$; (b) $\mu_{B}=300 \mathrm{MeV}$; (c) $\mu_{B}=600 \mathrm{MeV}$. Contributions of mesons (blue dotted line) and baryons (brown dashed line) are shown separately. With increasing baryon chemical potential, the baryon contribution to the total $\sigma^{e l}$ increases and the mesonic contribution decreases.

increases with temperature, reaching a maximum value, and eventually decreases at a higher temperature. This behavior of the normalized Hall conductivity with temperature is due to the convolution of multiple factors, e.g., the relaxation time, magnetic field, cyclotron frequency, and $1 / T^{2}$ dependence, similar to the behavior of electrical conductivity for a nonvanishing magnetic field.

In Fig. 6 we plot the variation of the normalized Hall conductivity $\sigma^{H} / T$ with temperature for different values of the baryon chemical potential at $B=0.05 \mathrm{GeV}^{2}$. As may be noted from this figure, for a smaller chemical potential the Hall conductivity is smaller. This is due to the fact that for a finite Hall conductivity the imbalance between the number of particles and antiparticles is required. With an increase in the baryon chemical potential, the number density of particles is significantly larger than that of antiparticles, leading to a nonvanishing Hall current. Again the nonmonotonic behavior of normalized Hall conductivity with temperature for a specific value of the magnetic field is similar to Fig. 5.

Finally, in order to connect our results to heavy-ion collision experiments, we estimate the electrical conductivity and Hall conductivity as a function of beam energy $\sqrt{s}$ along the chemical freeze-out curve. Figure 7 shows the variation of $\sigma^{e l} / T$ and $\sigma^{H} / T$ with center of mass energy $\sqrt{s}$ for different values of the magnetic field. The dependence of $\sigma^{e l} / T$ and $\sigma^{H} / T$ with the center of mass energy $\sqrt{s}$ is extracted using the statistical thermal model description of the particle yield at various $\sqrt{s}$ [97]. Under the assumption that the freeze-out parameters are independent of the magnetic field, one can use the fitted freeze-out 


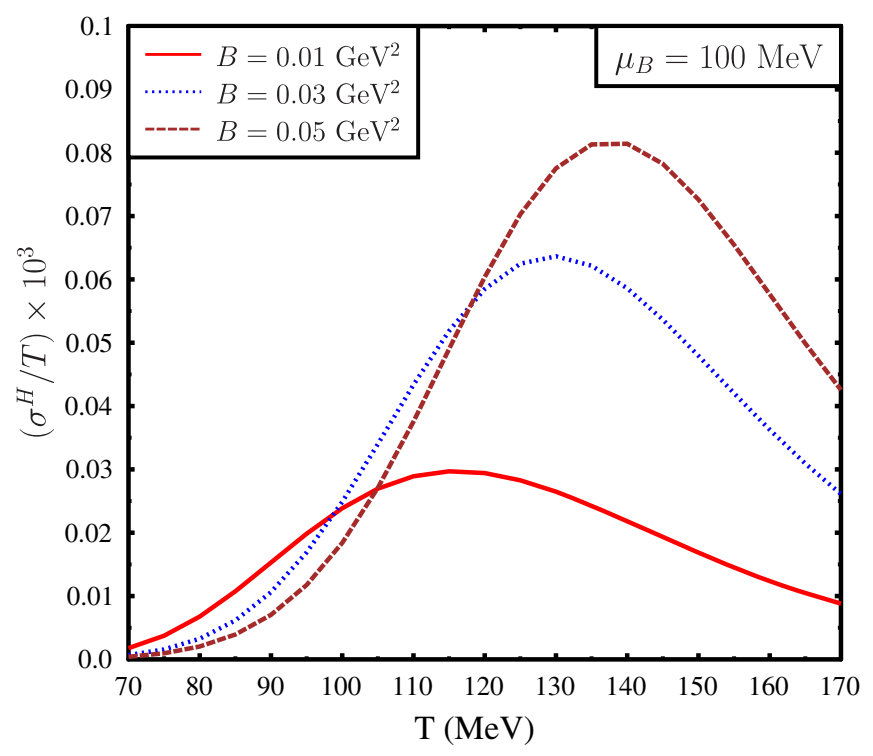

FIG. 5. Variation of normalized Hall conductivity $\sigma^{H} / T$ with temperature for various values of the magnetic field at $\mu_{B}=100 \mathrm{MeV}$. The red solid line, blue dotted line, and brown dashed line represent results for $B=0.01 \mathrm{GeV}^{2}, B=0.03 \mathrm{GeV}^{2}$, and $B=0.05 \mathrm{GeV}^{2}$, respectively. The Hall conductivity shows a nonmonotonic behavior with respect to the dependence on magnetic field. For low temperature the Hall conductivity decreases with an increase in magnetic field, while for higher temperature the Hall conductivity increases with magnetic field.

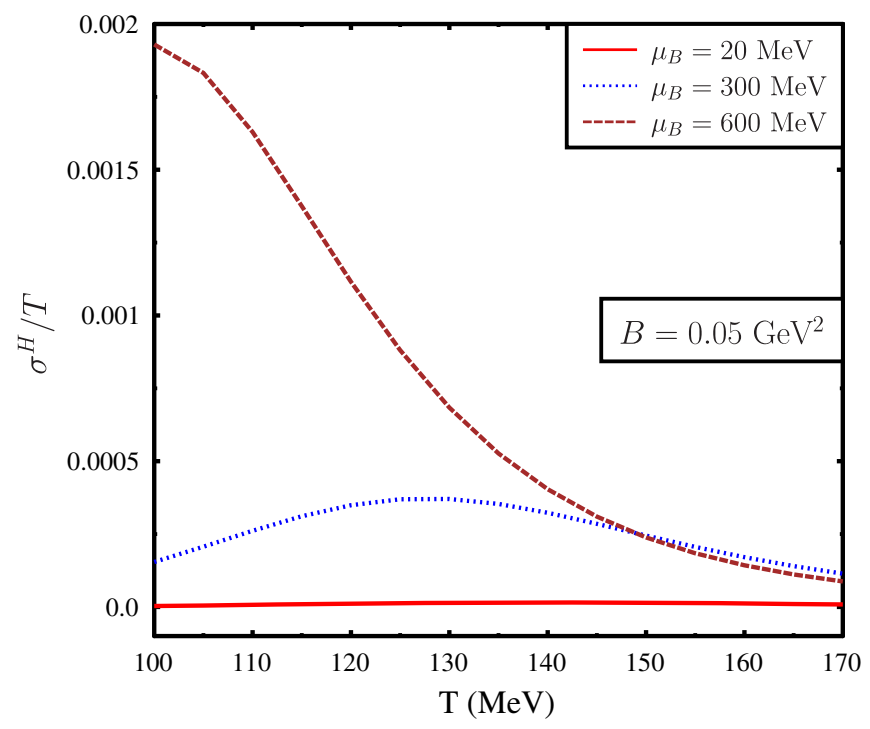

FIG. 6. Variation of normalized Hall conductivity $\sigma^{H} / T$ with temperature for various values of baryon chemical potential at $B=0.05 \mathrm{GeV}^{2}$. The red solid line, blue dotted line, and brown dashed line represent results for $\mu_{B}=20 \mathrm{MeV}, \mu_{B}=300 \mathrm{MeV}$, and $\mu_{B}=600 \mathrm{MeV}$, respectively. Due to the large number density of baryons at finite baryon chemical potential, Hall conductivity increases with increasing baryon chemical potential. temperature and baryon chemical potential as given by $T\left(\mu_{B}\right)=a-b \mu_{B}^{2}-c \mu_{B}^{4}$, with $a=0.166 \pm 0.002 \mathrm{GeV}$, $b=0.139 \pm 0.016 \mathrm{GeV}^{-1}, c=0.053 \pm 0.021 \mathrm{GeV}^{-3}$, and $\mu_{B}(\sqrt{s})=d /(1+e \sqrt{s})$, with $d=1.308 \pm 0.028 \mathrm{GeV}$, $e=0.273 \pm 0.008 \mathrm{GeV}^{-1}$, respectively [97]. In this investigation, we have considered the central values of the fitting parameters. The left plot and right plot in Fig. 7 show the variation of $\sigma^{e l} / T$ and $\sigma^{H} / T$ with the center of mass energy $\sqrt{s}$, respectively, for various values of the magnetic field. From this figure, it is clear that with an increasing magnetic field $\sigma^{e l} / T$ decreases and $\sigma^{H} / T$ increases. The variation of $\sigma^{e l} / T$ and $\sigma^{H} / T$ with $\sqrt{s}$ for a given magnetic field is nontrivial. At zero magnetic field $\sigma^{e l} / T$ decreases with increasing temperature as well as increasing baryon chemical potential. Now with a decreasing center of mass energy, the freeze-out temperature decreases but the freeze-out baryon chemical potential increases. Hence one has to consider the effect of both temperature and baryon chemical potential to understand the variation of $\sigma^{e l} / T$ with $\sqrt{s}$. Note that at finite magnetic field the variation of $\sigma^{e l} / T$ and $\sigma^{H} / T$ with temperature and chemical potential is more complicated than the case when the magnetic field is zero. Hence the variation of $\sigma^{e l} / T$ and $\sigma^{H} / T$ along the freeze-out curve is rather convoluted.

In Fig. 7 we have considered values of the magnetic field ranging from $B=0.001 \mathrm{GeV}^{2}$ to $B=0.04 \mathrm{GeV}^{2}$ for different center of mass energy $(\sqrt{s})$. Let us note that for RHIC the center of mass energy is $\sqrt{s}=200 \mathrm{GeV}$ and the estimated maximum magnetic field is of the order of $B=0.04 \mathrm{GeV}^{2}$ [14]. For this value of the magnetic field and the collision energy from Fig. 7 we get that the value of normalized Hall conductivity $\left(\sigma_{H} / T\right)$ for RHIC is of the order of $10^{-5}$ and the normalized electrical conductivity is of the order of $10^{-3}$. On the other hand for relatively low energy collisions, e.g., FAIR, the collision energy $E_{\text {lab }} \sim 10 \mathrm{~A} \mathrm{GeV}$ and the estimated maximum value of the magnetic field is of the order of $10^{-3} \mathrm{GeV}^{2}$ [14,98]. For this value of the magnetic field and the collision energy relevant for FAIR, the value of normalized Hall conductivity $\left(\sigma_{H} / T\right)$ is of the order of $10^{-4}$ and the normalized electrical conductivity is of the order of $10^{-2}$.

\section{CONCLUSIONS}

In this investigation, we have estimated the electrical $\left(\sigma^{e l}\right)$ and Hall conductivity $\left(\sigma^{H}\right)$ of a hot and dense hadron gas in the presence of an external magnetic field. We have estimated the electrical and Hall conductivity by solving the Boltzmann transport equation in the presence of the external electromagnetic field within the framework of a relaxation time approximation. It is important to note that the relaxation time approximation is valid when the external field is not strong. This assumption is valid in 

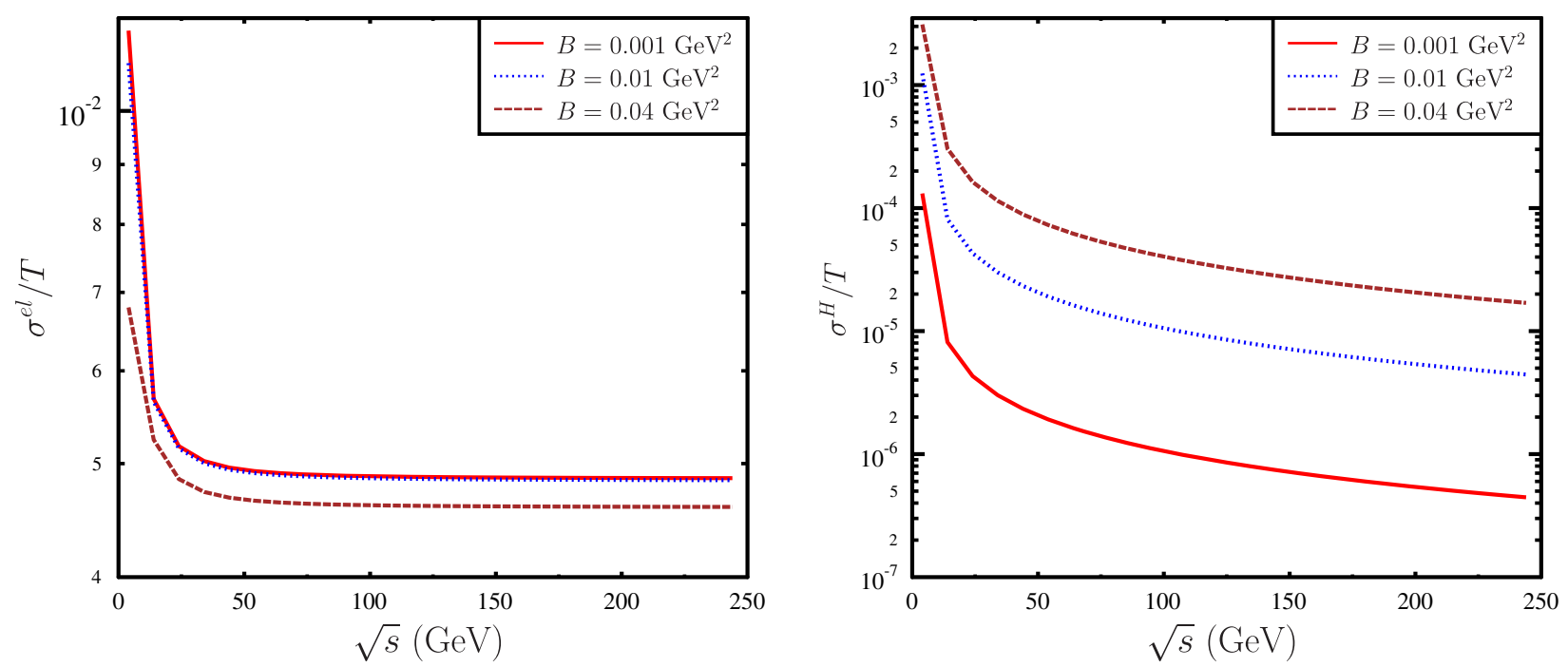

FIG. 7. Left: Variation of normalized electrical conductivity $\sigma^{e l} / T$ with center of mass energy along the chemical freeze-out curve for different values of the magnetic field. Right: Variation of normalized Hall conductivity $\sigma^{H} / T$ with center of mass energy ( $\sqrt{s}$ ) along the chemical freeze-out curve for different values of the magnetic field. With increasing magnetic field $\sigma^{e l} / T$ decreases and $\sigma^{H} / T$ increases.

the case of a hadron gas because at the freeze-out time, the strength of the magnetic field is relatively smaller than the initial magnetic field in heavy-ion collisions. Also, in this case, we have not considered the Landau quantization of the charged particles or the magnetic field dependent dispersion relation due to a relatively smaller magnetic field.

At vanishing magnetic field our results are similar to the previous results [19] as shown in Figs. 1 and 2. In the absence of a magnetic field $(B=0)$, pions are the dominant contributors in the electrical conductivity. With increasing temperature $(T)$ and chemical potential $\left(\mu_{B}\right)$ the relaxation time of the pion decreases. This gives rise to decreasing electrical conductivity with increasing temperature and baryon chemical potential. However, this situation is more complicated in the presence of a magnetic field. With increasing magnetic field $\sigma^{e l}$ decreases. This is because in the presence of a magnetic field, charged particles experience Lorentz force and they move in a direction transverse to the electric field and magnetic field. Since the baryons are heavier than the mesons, specifically pions, baryonic contributions can be important at nonzero magnetic field. With increasing chemical potential more baryons contribute to the electrical conductivity. Hence with increased chemical potential electrical conductivity increases. Variation of electrical conductivity in the presence of a magnetic field with temperature is rather complicated. Variation of $\sigma^{e l}$ with temperature is a convolution of various factors, e.g., temperature-dependent relaxation time, cyclotron frequency of different hadrons, and also the distribution functions. Generically with increasing temperature $\sigma^{e l}$ first increases, becomes maximum at intermediate temperature, and finally decreases.
Unlike electrical conductivity $\sigma^{e l}$, at zero baryon chemical potential Hall conductivity vanishes. In a condensed matter system Hall conductivity can be nonzero because in this case either electrons or holes are dominant charge carriers. However, in the case of pair plasma, the number density and mobility of particles and antiparticles are the same. Thus the Hall currents due to particles and antiparticles exactly cancel each other. This situation is similar to the case of a hot hadron gas when the baryon chemical potential $\mu_{B}$ is zero. Mesons do not contribute to the Hall conductivity. Only at finite baryon chemical potential, due to the difference in the numbers of baryons and antibaryons, one gets a nonvanishing Hall conductivity. It is obvious that with increasing baryon chemical potential the Hall conductivity should increase due to the increase in the number density of baryons. This is exactly what we get as a numerical result shown in Fig. 6. On the other hand, the dependence of the Hall conductivity on the magnetic field is nonmonotonic. While it increases with magnetic field for higher temperature, it decreases with magnetic field at lower temperature, which is evident from Fig. 5. This is due to the dependence of the Hall conductivity on cyclotron frequency and relaxation time as given in Eq. (19). It is important to mention that Hall conductivity depends upon the external magnetic field as well as on the baryon chemical potential. It is clear that for a given magnetic field Hall conductivity is less for a small baryon chemical potential. Also with increasing magnetic field Hall conductivity increases for a nonzero baryon chemical potential. We find that the value of normalized Hall conductivity is relatively smaller in the RHIC with respect to low energy collisions at FAIR. The value of normalized Hall conductivity $\left(\sigma^{H} / T\right)$ for the maximum value of a magnetic field at the RHIC and FAIR is of the order of $10^{-5}$ and $10^{-4}$, respectively. 


\section{ACKNOWLEDGMENTS}

R. K. M. would like to thank the Theoretical Physics Division of Physical Research Laboratory, Ahmedabad, for support and local hospitality for her visit, during which this problem was initiated. Also, R. K. M. would like to thank Basanta K. Nandi and Sadhana Dash for constant support and encouragement.
[1] U. W. Heinz and R. Snellings, Annu. Rev. Nucl. Part. Sci. 63, 123 (2013).

[2] P. Romatschke and U. Romatschke, Phys. Rev. Lett. 99, 172301 (2007).

[3] P. K. Kovtun, D. T. Son, and A. O. Starinets, Phys. Rev. Lett. 94, 111601 (2005).

[4] S. Gavin, Nucl. Phys. A435, 826 (1985).

[5] A. Hosoya and K. Kajantie, Nucl. Phys. B250, 666 (1985).

[6] A. Dobado and J. M. Torres-Rincon, Phys. Rev. D 86, 074021 (2012).

[7] C. Sasaki and K. Redlich, Phys. Rev. C 79, 055207 (2009).

[8] C. Sasaki and K. Redlich, Nucl. Phys. A832, 62 (2010).

[9] F. Karsch, D. Kharzeev, and K. Tuchin, Phys. Lett. B 663, 217 (2008).

[10] S. I. Finazzo, R. Rougemont, H. Marrochio, and J. Noronha, J. High Energy Phys. 02 (2015) 051.

[11] A. Wiranata and M. Prakash, Nucl. Phys. A830, 219C (2009).

[12] S. Jeon and L. Yaffe, Phys. Rev. D 53, 5799 (1996).

[13] D. E. Kharzeev, L. D. McLerran, and H. J. Warringa, Nucl. Phys. A803, 227 (2008).

[14] V. Skokov, A. Yu. Illarionov, and V. Toneev, Int. J. Mod. Phys. A 24, 5925 (2009).

[15] Strongly Interacting Matter in Magnetic Field, edited by D. Kharzeev, K. Landsteiner, A. Schmitt, and H. Yee, Lecture Notes in Physics Vol. 871 (Springer-Verlag, Berlin, Heidelberg, 2013).

[16] G. Inghirami, L. D. Zanna, A. Beraudo, M. H. Moghaddam, F. Becattini, and M. Bleicher, Eur. Phys. J. C 76, 659 (2016).

[17] A. Das, S. S. Dave, P. S. Saumia, and A. M. Srivastava, Phys. Rev. C 96, 034902 (2017).

[18] K. Tuchin, Phys. Rev. C 83, 017901 (2011); 82, 034904 (2010).

[19] M. Greif, C. Greiner, and G. S. Denicol, Phys. Rev. D 93, 096012 (2016).

[20] M. Greif, I. Bouras, C. Greiner, and Z. Xu, Phys. Rev. D 90, 094014 (2014).

[21] A. Puglisi, S. Plumari, and V. Greco, Phys. Lett. B 751, 326 (2015).

[22] A. Puglisi, S. Plumari, and V. Greco, Phys. Rev. D 90, 114009 (2014).

[23] W. Cassing, O. Linnyk, T. Steinert, and V. Ozvenchuk, Phys. Rev. Lett. 110, 182301 (2013).

[24] T. Steinert and W. Cassing, Phys. Rev. C 89, 035203 (2014).

[25] G. Aarts, C. Allton, A. Amato, P. Giudice, S. Hands, and J.-I. Skullerud, J. High Energy Phys. 02 (2015) 186.

[26] G. Aarts, C. Allton, J. Foley, S. Hands, and S. Kim, Phys. Rev. Lett. 99, 022002 (2007).
[27] A. Amato, G. Aarts, C. Allton, P. Giudice, S. Hands, and J.-I. Skullerud, Phys. Rev. Lett. 111, 172001 (2013).

[28] S. Gupta, Phys. Lett. B 597, 57 (2004).

[29] Y. Burnier and M. Laine, Eur. Phys. J. C 72, 1902 (2012).

[30] H.-T. Ding, A. Francis, O. Kaczmarek, F. Karsch, E. Laermann, and W. Soeldner, Phys. Rev. D 83, 034504 (2011).

[31] O. Kaczmarek and M. Muller, Proc. Sci, LATTICE2013 (2014) 175.

[32] S.-X. Qin, Phys. Lett. B 742, 358 (2015).

[33] R. Marty, E. Bratkovskaya, W. Cassing, J. Aichelin, and H. Berrehrah, Phys. Rev. C 88, 045204 (2013).

[34] D. Fernandez-Fraile and A. Gomez Nicola, Phys. Rev. D 73, 045025 (2006).

[35] M. Greif, J. A. Fotakis, G. S. Denicol, and C. Greiner, Phys. Rev. Lett. 120, 242301 (2018).

[36] M. Prakash, M. Prakash, R. Venugopalan, and G. Welke, Phys. Rep. 227, 321 (1993).

[37] A. Wiranata and M. Prakash, Phys. Rev. C 85, 054908 (2012).

[38] P. Chakraborty and J. I. Kapusta, Phys. Rev. C 83, 014906 (2011).

[39] A. S. Khvorostukhin, V. D. Toneev, and D. N. Voskresensky, Nucl. Phys. A845, 106 (2010).

[40] S. Plumari, A. Paglisi, F. Scardina, and V. Greco, Phys. Rev. C 86, 054902 (2012).

[41] M. I. Gorenstein, M. Hauer, and O. N. Moroz, Phys. Rev. C 77, 024911 (2008).

[42] J. Noronha-Hostler, J. Noronha, and C. Greiner, Phys. Rev. C 86, 024913 (2012).

[43] S. K. Tiwari, P. K. Srivastava, and C. P. Singh, Phys. Rev. C 85, 014908 (2012).

[44] S. Ghosh, A. Lahiri, S. Majumder, R. Ray, and S. K. Ghosh, Phys. Rev. C 88, 068201 (2013).

[45] R. Lang, N. Kaiser, and W. Weise, Eur. Phys. J. A 51, 127 (2015).

[46] S. Ghosh, G. Krein, and S. Sarkar, Phys. Rev. C 89, 045201 (2014).

[47] A. Wiranata, V. Koch, M. Prakash, and X. N. Wang, J. Phys. Conf. Ser. 509, 012049 (2014).

[48] A. Wiranata, M. Prakash, and P. Chakraborty, Central Eur. J. Phys. 10, 1349 (2012).

[49] A. Tawfik and M. Wahba, Ann. Phys. (Amsterdam) 522, 849 (2010).

[50] J. Noronha-Hostler, J. Noronha, and C. Greiner, Phys. Rev. Lett. 103, 172302 (2009).

[51] G. Kadam and H. Mishra, Nucl. Phys. A934, 133 (2015).

[52] G. Kadam, Mod. Phys. Lett. A 30, 1550031 (2015).

[53] S. Ghosh, Int. J. Mod. Phys. A 29, 1450054 (2014). 
[54] N. Demir and A. Wiranata, J. Phys. Conf. Ser. 535, 012018 (2014).

[55] S. Ghosh, Phys. Rev. C 90, 025202 (2014).

[56] J.-B. Rose, J. M. Torres-Rincon, A. Schfer, D. R. Oliinychenko, and H. Petersen, Phys. Rev. C 97, 055204 (2018).

[57] C. Wesp, A. El, F. Reining, Z. Xu, I. Bouras, and C. Greiner, Phys. Rev. C 84, 054911 (2011).

[58] Moritz Greif, Ioannis Bouras, Carsten Greiner, and Zhe Xu, Phys. Rev. D 90, 094014 (2014).

[59] S. A. Bass et al., Prog. Part. Nucl. Phys. 41, 255 (1998).

[60] G. Kadam and H. Mishra, Phys. Rev. C 92, 035203 (2015).

[61] R. K. Mohapatra, H. Mishra, S. Dash, and B. K. Nandi, arXiv:1901.07238.

[62] P. Singha, A. Abhishek, G. Kadam, S. Ghosh, and H. Mishra, J. Phys. G 46, 015201 (2019).

[63] A. Abhishek, H. Mishra, and S. Ghosh, Phys. Rev. D 97, 014005 (2018).

[64] J. R. Bhatt, A. Das, and H. Mishra, Phys. Rev. D 99, 014015 (2019).

[65] G. S. Denicol, H. Niemi, I. Bouras, E. Molnar, Z. Xu, D. H. Rischke, and C. Greiner, Phys. Rev. D 89, 074005 (2014).

[66] J. I. Kapusta and J. M. Torres-Rincon, Phys. Rev. C 86, 054911 (2012).

[67] K. Tuchin, Phys. Rev. C 88, 024911 (2013).

[68] K. Tuchin, Adv. High Energy Phys. 2013, 1 (2013).

[69] B. Feng, Phys. Rev. D 96, 036009 (2017).

[70] C. Hamaguchi, Basic Semiconductor Physics (Springer, Berlin, Heidelberg, 2010).

[71] A. Kandus and C. G. Tsagas, Mon. Not. R. Astron. Soc. 385, 883 (2008).

[72] E. G. Blackman and G. B. Field, Phys. Rev. Lett. 71, 3481 (1993).

[73] N. Bessho and A. Bhattacharjee, Phys. Plasmas 14, 056503 (2007).

[74] See, https://www.gsi.de/en/researchaccelerators/fair.htm.

[75] See, http://nica.jinr.ru.

[76] P. Braun-Munzinger, K. Redlich, and J. Stachel, arXiv:nuclth/0304013.

[77] A. Andronic, P. Braun-Munzinger, and J. Stachel, Nucl. Phys. A772, 167 (2006).
[78] P. Braun-Munzinger, D. Magestro, K. Redlich, and J. Stachel, Phys. Lett. B 518, 41 (2001); J. Cleymans and K. Redlich, Phys. Rev. C 60, 054908 (1999); F. Becattini, J. Cleymans, A. Keränen, E. Suhonen, and K. Redlich, Phys. Rev. C 64, 024901 (2001); J. Cleymans, B. Kampfer, M. Kaneta, S. Wheaton, and N. Xu, Phys. Rev. C 71, 054901 (2005); A. Andronic, P. Braun-Munzinger, and J. Stachel, Phys. Lett. B 673, 142 (2009).

[79] R. Dashen, S. Ma, and H. J. Bernstein, Phys. Rev. 187, 345 (1969).

[80] R. Dashen and R. Rajaraman, Phys. Rev. D 10, 694 (1974).

[81] F. Karsch, K. Redlich, and A. Tawfik, Phys. Lett. B 571, 67 (2003).

[82] P. Braun-Munzinger, V. Koch, T. Schafer, and J. Stachel, Phys. Rep. 621, 76 (2016).

[83] M. Nahrgang, M. Bluhm, P. Alba, R. Bellwied, and C. Ratti, Eur. Phys. J. C 75, 573 (2015).

[84] A. Bhattacharyya, S. Das, S. K. Ghosh, R. Ray, and S. Samanta, Phys. Rev. C 90, 034909 (2014).

[85] P. Garg, D. K. Mishra, P. K. Netrakanti, B. Mohanty, A. K. Mohanty, B. K. Singh, and N. Xu, Phys. Lett. B 726, 691 (2013).

[86] A. Bazavov et al., Phys. Rev. D 86, 034509 (2012).

[87] V. V. Begun, M. I. Gorenstein, M. Hauer, V. P. Konchakovski, and O. S. Zozulya, Phys. Rev. C 74, 044903 (2006).

[88] R. K. Mohapatra, Phys. Rev. C 99, 024902 (2019).

[89] D. H. Rischke, M. I. Gorenstein, H. Stocker, and W. Greiner, Z. Phys. C 51, 485 (1991).

[90] G. Kadam, H. Mishra, and L. Thakur, Phys. Rev. D 98, 114001 (2018).

[91] A. Majumder and B. Muller, Phys. Rev. Lett. 105, 252002 (2010).

[92] P. Deb, G. Kadam, and H. Mishra, Phys. Rev. D 94, 094002 (2016).

[93] P. Gondolo and G. Gelmini, Nucl. Phys. B360, 145 (1991).

[94] C. Amsler et al. (Particle Data Group), Phys. Lett. B 667, 1 (2008).

[95] M. Albright, J. Kapusta, and C. Young, Phys. Rev. C 90, 024915 (2014).

[96] P. Braun-Munzinger, I. Heppe, and J. Stachel, Phys. Lett. B 465, 15 (1999).

[97] J. Cleymans, H. Oeschler, K. Redlich, and S. Wheaton, Phys. Rev. C 73, 034905 (2006).

[98] P Senger, J. Phys.: Conf. Ser. 798, 012062 (2017). 